phys. stat. sol. (b) 61, 533 (1974)

Subject classification : $6 ; 20.1 ; 22.7$

Physikalisches Institut der Universität Würzburg

\title{
Optically Active Lattice Vibrations in $\mathrm{Bi}_{2} \mathrm{Se}_{3}$
}

\author{
By \\ H. KöHLER and C. R. BECKER
}

The preparation of $\mathrm{Bi}_{2} \mathrm{Se}_{3}$ single crystals with low free carrier densities allowed an investigation of the lattice vibrations to be carried out from the reflectivity of cleavage planes at nearly normal incidence of the radiation $(\boldsymbol{E}, \boldsymbol{c})$. The experimental results can be explained with two classical oscillators, whose eigenfrequencies occur at 92 and $69.5 \mathrm{~cm}^{-1}$. The static dielectric constant was determined to be $100 \pm 10$ for $\boldsymbol{E} \perp \boldsymbol{c}$.

Die Präparation von $\mathrm{Bi}_{2} \mathrm{Se}_{3}$-Einkristallen mit niedriger Konzentration freier Ladungsträger erlaubte die Untersuchung der Gitterschwingungen aus dem Reflexionsvermögen von Spaltflächen bei nahezu senkrechter Inzidenz der Strahlung $(\boldsymbol{E} \perp \boldsymbol{c})$. Die MeBergebnisse können mit zwei klassischen Dispersionsoszillatoren erklärt werden, deren Eigenfrequenzen bei 92 und $69,5 \mathrm{~cm}^{-1}$ liegen. Die statische Dielektrizitätskonstante wurde zu $100 \pm 10$ für $\boldsymbol{E} \perp \boldsymbol{c}$ bestimmt.

\section{Introduction}

Bismuth selenide crystallizes in a layer-type structure belonging to the space group $R \overline{3} \mathrm{~m}$, as is also the case for the semiconductors $\mathrm{Bi}_{2} \mathrm{Te}_{3}, \mathrm{Sb}_{2} \mathrm{Te}_{3}$, and their solid solutions. However, $\mathrm{Bi}_{2} \mathrm{Se}_{3}$ is more difficult to prepare than $\mathrm{Bi}_{2} \mathrm{Te}_{3}$. The non-stoichiometric composition of the crystals normally produces high freecarrier concentrations.

A reduction in free-carrier concentration by doping with impurity atoms is possible in $\mathrm{Bi}_{2} \mathrm{Te}_{3}$, but not to a significant extent in $\mathrm{Bi}_{2} \mathrm{Se}_{3}$ and $\mathrm{Sb}_{2} \mathrm{Te}_{3}$. Low carrier densities of about $10^{17} \mathrm{~cm}^{-3}$ were achieved in $\mathrm{Bi}_{2} \mathrm{Te}_{3}$ by doping with iodine [1], respectively excess tellurium in the melt. Whereas, to our knowledge, the lowest free-carrier concentration achieved in $\mathrm{p}-\mathrm{Sb}_{2} \mathrm{Te}_{3}$ is of the order of magnitude of some $10^{19} \mathrm{~cm}^{-3}$. Even though the doping on n-type, as-grown $\mathrm{Bi}_{2} \mathrm{Se}_{3}$ with impurity atoms is ineffective, a higher selenium content in the melt has been used to obtain carrier densities down to $4 \times 10^{16}$ electrons $/ \mathrm{cm}^{3}$.

The smaller the free-carrier concentration the smaller their contribution to the optical constants, which more readily allows the influence of the optically active lattice vibrations to be ascertained. However, the cleavage - normal to the trigonal $c$-axis - of crystals with low carrier concentrations is difficult in order to achieve high quality optical surfaces. A mechanical or chemical polish of surfaces with good optical quality is impossible at the present. Since reflection measurements are most conveniently carried out under nearly normal incident conditions, the electric field vector $\boldsymbol{E}$ is parallel to the crystal surface and therefore perpendicular to the trigonal axis $(\boldsymbol{E} \perp \boldsymbol{c})$ for cleavage planes under experiment. Thus, only optically active modes which involve movements of the atoms normal to the $c$-axis may be observed with normal incident radiation, i.e. the conditions of the present measurements.

The electronic band structure of $n$ - and p-type $\mathrm{Bi}_{2} \mathrm{Te}_{3}$ has been well known for a long time [ 2 to 7 ] because of its easier preparation. Besides that, investigations of $\mathrm{Bi}_{2} \mathrm{Te}_{3}$ advanced more rapidly as a result of its outstanding thermo- 
electric properties which made the crystals more attractive for thermoelectric cooling. Because $\mathrm{Bi}_{2} \mathrm{Se}_{3}$ has the same symmetry and almost identical lattice spacings, similar properties were expected. It was found, however, that the electronic properties of the conduction bands differ appreciably from each other $[8,9]$. Valence band parameters in $\mathrm{Bi}_{2} \mathrm{Se}_{3}$ are still unknown, because of the difficulty incurred in preparing p-type material.

The elastic constants for $\mathrm{Bi}_{2} \mathrm{Te}_{3}$ [10] and specific heat values for $\mathrm{Bi}_{2} \mathrm{Te}_{3}$ and $\mathrm{Bi}_{2} \mathrm{Se}_{3}$ [11] have been determined. From the specific heat data, Debye temperatures were calculated for temperatures between 1.4 and $90 \mathrm{~K}$. From a comparison of Debye temperatures near absolute zero, $0_{0}$, almost identical force constants for $\mathrm{Bi}_{2} \mathrm{Te}_{3}$ and $\mathrm{Bi}_{2} \mathrm{Se}_{3}$ were concluded [11], and hence, correspondingly similar lattice dynamies were expected. The Debye temperature at high temperatures, which is equal to 200 and $154 \mathrm{~K}$ for $\mathrm{Bi}_{2} \mathrm{Se}_{3}$ and $\mathrm{Bi}_{2} \mathrm{Te}_{3}$, respectively, permits one to estimate averaged longitudinal optical frequencies of 139 and $107 \mathrm{~cm}^{-1}$, respectively. Thus slightly higher eigenfrequencies are expected for $\mathrm{Bi}_{2} \mathrm{Se}_{3}$ in comparison with $\mathrm{Bi}_{2} \mathrm{Te}_{3}$. However, in spite of the above mentioned similarities, the lattice vibration contributions to the long wavelength dielectric constant may differ in the two cases because of a different degree of polarizability of the crystal lattices.

A rigid ion calculation involving only first and second nearest neighbour forces [10] has been made using measurements of the elastic constants of $\mathrm{Bi}_{2} \mathrm{Te}_{3}$ between 4.2 and $300 \mathrm{~K}$. However, the departure of the experimental elastic constants for $\mathrm{Bi}_{2} \mathrm{Te}_{3}$ from those obtained from the Debye theory already at $T \approx$ $\approx \theta / 300$ indicates the presence of either strong polarizabilities or a significant contribution from atoms more distant than first and second nearest neighbours. The extraordinarily high value for the static dielectric constant of $\mathrm{Bi}_{2} \mathrm{Te}_{3}[1]$ in comparison with $\mathrm{Bi}_{2} \mathrm{Se}_{3}$ supports the former.

The eigenfrequencies calculated for $\mathrm{Bi}_{2} \mathrm{Te}_{3}$ using the above mentioned lattice model [10], were only of the correct order of magnitude [1] as one would also expect to be the case for a similar calculation for $\mathrm{Bi}_{2} \mathrm{Se}_{3}$.

The primitive unit cell contains five atoms and, therefore, 15 degrees of freedom are allowed whose irreducible representation at $k=0$ is

$$
\Gamma_{\mathrm{i}}^{\prime}=2 \mathrm{~A}_{1 \mathrm{~g}}+3 \mathrm{~A}_{1 \mathrm{u}}+2 \mathrm{E}_{\mathrm{g}}+3 \mathrm{E}_{\mathrm{u}} \text {, }
$$

where $A_{1 u}+E_{u}$ are the acoustic modes. The remaining $2 A_{1 u}+2 E_{u}$ are optically active for $\boldsymbol{E} \| \boldsymbol{c}$ and $\boldsymbol{E} \perp \boldsymbol{c}$, respectively. The second-order symmetric tensor representation $2 \mathrm{~A}_{1 \mathrm{~g}}+2 \mathrm{E}_{\mathrm{g}}$ yields the Raman-active lattice vibrations.

\section{Experimental}

Experimentally, several crystals with low carrier densities were investigated. The reflectivity of cleavage planes was measured at nearly normal incidence with

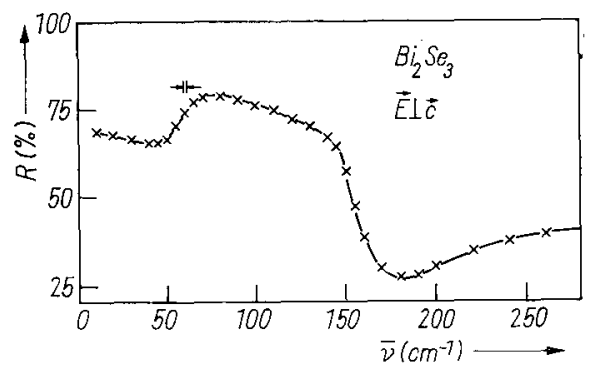

Fig. 1. Reflectivity of $n-\mathrm{Bi}_{2} \mathrm{Se}_{3}$ (single crystal with a carrier density of $5 \times 10^{17} \mathrm{~cm}^{-3}$ ) versus frequency at nearly normal incidence of the radiation, where $\boldsymbol{E}_{-}$_ $_{\mathbf{c}}$. The optical surface of the sample is a cleavage plane 
Fig. 2. Kramers-Kronig analysis of the reflectivity data in Fig. 1. The real part of the dielectric constant $n^{2}-k^{2}$ (solid line) and the absorption $n K=4 \pi n k / \lambda$ (solid line) is plotted versus the wavelength. The theoretical results from (1) and (2) using the parameter values given in (3) are drawn as broken lines (----)

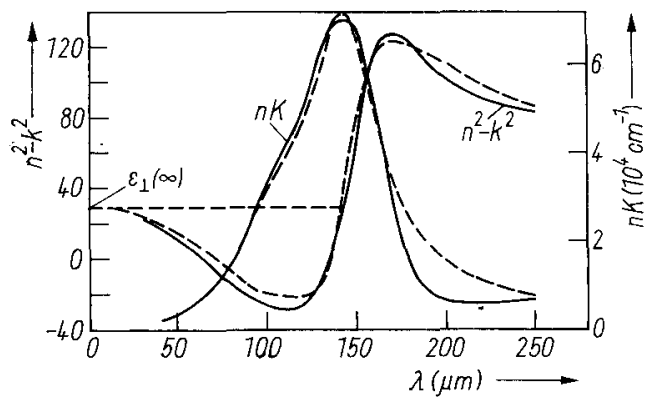

$\boldsymbol{E} \perp \boldsymbol{c}$ at $300 \mathrm{~K}$ with a Polytec FIR 30 Fourier spectrometer. The results for one sample with a free earrier concentration of $5 \times 10^{17} \mathrm{~cm}^{-3}$ are shown in Fig. 1 for frequencies between 10 and $280 \mathrm{~cm}^{-1}$. The experimental resolution is given. There is a steep rise in the reflectivity $R$ from 170 to $145 \mathrm{~cm}^{-1}$, which is followed by a more gradual increase of $R$ down to $75 \mathrm{~cm}^{-1}$. The drop of $R$ from 70 to $50 \mathrm{~cm}^{-1}$ indicates the position of one optical mode eigenfrequency. The increase in the reflectivity below $40 \mathrm{~cm}^{-1}$ for decreasing frequency is caused by free carriers. The steep part of the curve near $160 \mathrm{~cm}^{-1}$ is shifted to higher frequencies for crystals with larger free carrier concentrations but is not influenced by crystals with lower free carrier concentrations. Thus the contribution of free carriers is almost negligible in the results shown in Fig. 1.

\section{Analysispand Discussion}

In order to evaluate the measurements a Kramers-Kronig analysis was performed, taking into account known reflectivity data for higher wave numbers and approximating the $R(\tilde{v})$ dependence by free carrier influence below $20 \mathrm{~cm}^{-1}$.

The results of this calculation are given in Fig. 2, where the real part of the dielectric constant $n^{2}-k^{2}$ and the absorption $n K(K=4 \pi k / \lambda$ absorption constant) are plotted versus the wavelength (unbroken curves). A maximum in the absorption is found at $\lambda=144 \mu \mathrm{m}$, where one of the $\mathrm{E}_{\mathrm{u}}$ eigenfrequencies occurs $\left(\tilde{v}_{\perp 1}=69.5 \mathrm{~cm}^{-1}\right)$. The unsymmetric shape of the absorption curve suggests the presence of a second lattice vibration, which is also expected from group theory.

We tried to fit theoretical calculations (broken curves in Fig. 2) to the Kramers-Kronig results, assuming a model with two independent classical oscillators for the lattice vibrations and using Drude's theory for the free carrier contributions $(\boldsymbol{E} \perp \boldsymbol{c})$ :

$$
\begin{gathered}
n^{2}-k^{2}=\varepsilon_{\perp}(\infty)+\sum_{i=1,2} \frac{\Delta \varepsilon_{\perp i}\left(\omega_{\perp i}^{2}-\omega^{2}\right) \omega_{\perp i}^{2}}{\left(\omega_{\perp i}^{2}-\omega^{2}\right)^{2}+\omega^{2} \gamma_{\perp i}^{2}}-\frac{N_{e}^{2}}{\varepsilon_{0} m^{*}} \frac{\tau^{2}}{\left(1+\omega^{2} \tau^{2}\right)}, \\
n K=\sum_{i=1,2} \frac{\Delta \varepsilon_{\perp i} \omega^{2} \omega_{\perp i}^{2} \gamma_{\perp i} / c}{\left(\omega_{\perp i}^{2}-\omega^{2}\right)^{2}+\omega^{2} \gamma_{\perp i}^{2}}+\frac{N_{e}^{2}}{\varepsilon_{0} m^{*} c} \frac{\tau}{\left(1+\omega^{2} \tau^{2}\right)} .
\end{gathered}
$$

Here $\omega_{\perp i}=2 \pi c \tilde{v}_{\perp i}$ are the $\mathrm{E}_{\mathrm{u}}$ frequencies, $\Delta \varepsilon_{\perp i}$ are the contributions of the classical oscillators to the dielectric constant, and $\gamma_{\perp i}$ describe the damping of the lattice vibrations. $N$ is the free carrier concentration, $m^{*}$ their susceptibility mass, and $\tau$ their relaxation time. The frequency $\omega$ of the radiation is related to the wave number $\tilde{\nu}$ and wavelength $\lambda$ by $\omega=2 \pi c \tilde{\nu}=2 \pi c / \lambda . m *$ is known from 
Shubnikov-de Haas investigations [9], $N$ was determined from the Hall constant, and $\tau$ could be estimated from transport coefficients.

The best fit (broken lines in Fig. 2) was achieved with the following parameters. We should mention here the failure of one classical oscillator to describe the experimental data.

$$
\left.\begin{array}{rl}
\tilde{v}_{\perp 1} & =69.5 \mathrm{~cm}^{-1}, \quad \Delta \varepsilon_{\perp 1}=43, \quad 2 \Delta \tilde{v}_{\perp 1}=22 \mathrm{~cm}^{-1}, \\
\tilde{v}_{\perp 2} & =92 \mathrm{~cm}^{-1}, \quad \Delta \varepsilon_{\perp 2}=24, \quad 2 \Delta \tilde{v}_{\perp 2}=55 \mathrm{~cm}^{-1}, \\
N & =5 \times 10^{17} \mathrm{~cm}^{-3}, \quad m^{*}=0.124 m_{0}, \quad \tau=7 \times 10^{-13} \mathrm{~s}, \quad \varepsilon_{\perp}(\infty)=29,
\end{array}\right\}
$$

where $\gamma=2 \Delta \omega=2 \pi c(2 \Delta \tilde{v}),(2 \Delta \tilde{v})$ being the width at half-maximum. Indeed, the free carrier contribution is not important. The static diclectric constant $\varepsilon_{\perp}(0)=\varepsilon_{\perp}(\infty)+\Delta \varepsilon_{\perp 1}+\Delta \varepsilon_{\perp 2}$ was found to be 96 . Taking into account experimental uncertainties, the quality of the crystal surface and errors introduced by the assumed classical model, the value $\varepsilon_{\perp}(0)=100-10$ is reasonable. Even, though the polarizability of $\mathrm{Bi}_{2} \mathrm{Se}_{3}$ is considerable, it is still much less than that of $\mathrm{Bi}_{2} \mathrm{Te}_{3}$, where $\varepsilon_{\perp}(0)=360 \pm 50$ was found $[1]$. This explains why acoustical phonon scattering plays such an important role in transport phenomena in $\mathrm{Bi}_{2} \mathrm{Te}_{3}$ down to $10 \mathrm{~K}$, and thus an influence of charged impuritics or defects is partially screened out by the polarizability of the host lattice. Thus the differences in transport phenomena in $\mathrm{Bi}_{2} \mathrm{Se}_{3}\lceil 12]$ and $\left.\mathrm{Bi}_{2} \mathrm{Te}_{3}\lceil\mathrm{l}\rfloor\right\rfloor$ can be explained by a much lower static dielectric constant with less sereening of the charged centres.

The damping constants, especially $\gamma_{\perp 2}$, are considerable. This indicates in the same way as for $\mathrm{Bi}_{2} \mathrm{Te}_{3}$ that the $\mathrm{Bi}_{2} \mathrm{Se}_{3}$ lattice has to contain a high concentration of lattice defects.

The results confirm that the lattice force constants of $\mathrm{Bi}_{2}{ }^{r} \mathrm{Tc}_{3}$ and $\mathrm{Bi}_{2} \mathrm{Sc}_{3}$ are approximately equal, but that the polarizability of the tellurium atom has to be much higher than that of the selenium atom in the corresponding compound.

Neglecting the contribution by free carricrs, $n^{2}-k^{2}=0$ occurs at about $149 \mathrm{~cm}^{-1}$. This may be compared to the value $k \theta_{\infty} /(h c)=139 \mathrm{~cm}^{-1}$ which was calculated from the Debye temperature $\theta_{\infty}=200 \mathrm{~K}$, and which is therefore sort of an average frequency, showing that also for $\boldsymbol{E}:\left(n^{2}-k^{2}=0\right.$ should occur at about $149 \mathrm{~cm}^{-1}$.

Neglecting the frec carrier contribution in $(1), n^{2}-k^{2}=0$ yiclds $\left(\tilde{\boldsymbol{v}}=\tilde{v}_{0}\right)$

$$
\begin{gathered}
\left(\varepsilon_{\perp}(\infty)+\Delta \varepsilon_{\perp 1}\right) \frac{\tilde{v}_{\perp 1}^{2}}{\tilde{v}_{0}^{2}}+\left(\varepsilon_{\perp}(\infty)+\Delta \varepsilon_{\perp 2}\right) \frac{\tilde{v}_{\perp}^{2}}{\tilde{v}_{0}^{2}}= \\
=\varepsilon_{\perp}(0) \frac{\tilde{v}_{\perp 1}^{2}}{\tilde{v}_{0}^{2}} \frac{v_{\perp 2}^{2}}{\tilde{v}_{0}^{2}}+\varepsilon_{\perp}(\infty),
\end{gathered}
$$

when the influence of damping is omitted. Upon inserting the above values in (4) quite a good agreement between the sides of the equation is found. This encourages one to use the same relation for the case $\boldsymbol{E} \| \boldsymbol{c}$, where $\tilde{v}_{0}$ should be equal or somewhat less than $149 \mathrm{~cm}^{-1}$. Taking into account that eigenfrequencies for $\boldsymbol{E} \| \boldsymbol{c}$ were calculated from the lattice model of Jenkins et al. [10] to be greater than for $\boldsymbol{E} \perp \boldsymbol{c}$ and in addition $\varepsilon_{\perp}(\infty) \approx \varepsilon_{\mid 1}(\infty)$ [14], a smaller static dielectric constant may be expected for $\boldsymbol{E} \| \boldsymbol{c}$ in comparison with $\boldsymbol{E} \perp \boldsymbol{c}$.

For the same reasons $\left(n^{2}-k^{2}=0\right.$ occurs at $\tilde{\boldsymbol{v}}_{0}=125 \mathrm{~cm}^{-1}$ for $\boldsymbol{E} \perp \boldsymbol{c}$ and $k \theta_{\infty} /(h c)=107 \mathrm{~cm}^{-1}$ ) and upon taking into consideration the values of $\varepsilon_{\perp}(\infty) \approx$ 
$\approx 80$ and $\varepsilon_{1}(\infty) \approx 50$ of $\mathrm{Bi}_{2} \mathrm{Te}_{3}[15]$ a strongly anisotropic static diclectric constant has to be assumed for $\mathrm{Bi}_{2} \mathrm{Te}_{3}$, which recently was proved experimentally $[16]$.

\section{Acknouledgement}

The financial support of the Deutsche Forschungsgemeinschaft is appreciated.

\section{References}

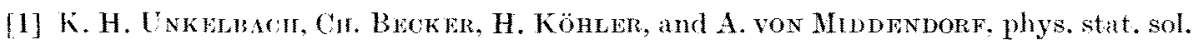
(b) 60, K41 (1973).

[2] J. R. Dtabbik, Progr. Semicond. 7, 45 (1963).

[3] H. J. Gor, smon, Materials Used in Semicondnctor Devices, Ed. (. A. Hocarth, Interscience Publishers, New York 1965 (p. 165).

[1] J. R. Drarbie, R. D. (Gnoves, and R. Wolfe, Proc Phys. Soc. (London) 71, 430 (1958).

[5] 1. (4. Austin, Proc. Phys. Soc. (London) 76, 169)(1960).

[6] R. 13. Matllinson, J. A. Raven, and R. W. URe, Phys. Rev. 175, 1049 (1968).

[7] A. von Midpen dorz and G. Landw Ehe, Solid State Commun. 11, 203 (1972).

[8] H. Kömer and (a. Lavoweme, phys. stat. sol. (b) 45, K109 (1971).

[9] H. Köntsk, phys. stat. sol. (b) 5s. 91 (1973).

[10] .J. O. Jenkins, J. A. Rayne, and R. W. Ure, Phys. Rev. B 5, 3171 (1972).

[11] (a. E. Shomake, J. A. Rayne, and R. W. Ure, Phys. Rev. 185, 1046 (1969).

[12] A. von MuDDENDORFF, H. Köhlem, and G. LandweIr, phys. stat. sol. (b) 57, 203 (1973).

[13] P. A. Walknk. Proce Phys. Soc 7ti, 113 (1960).

[14] H. Cobrecht and S. Sikeck, Z. Phys. פev, 93 (1969).

[15] R. Groth and P. SenNabel. J. Phys. Chem. Solids 2.), 1261 (1964).

[16] K. H. UNK HLBACH, private communication.

(Received August 23, 1973) 